\title{
Presence of ductal carcinoma in situ confers an improved prognosis for patients with T1NOMO invasive breast carcinoma
}

\section{A.F. Logullo ${ }^{1}$, A.B. Godoy², \\ M. Mourão-Neto ${ }^{3}$, \\ A.J.G. Simpson², \\ I.N. Nishimoto ${ }^{3}$ and M.M. Brentani ${ }^{1}$}

\author{
'Disciplina de O ncologia, Departamento de Radiologia, Faculdade de Medicina, \\ Universidade de São Paulo, São Paulo, SP, Brasil \\ ${ }^{2}$ Instituto Ludwig de Pesquisa sobre o Câncer, São Paulo, SP, Brasil \\ ${ }^{3}$ Hospital do Câncer A.C. Camargo, São Paulo, SP, Brasil
}

\author{
Correspondence \\ M.M. Brentani \\ Disciplina de Oncologia \\ Departamento de Radiologia \\ FM, USP \\ Av. Dr. Arnaldo, 455, Sala 4112 \\ 01246-903 São Paulo, SP \\ Brasil \\ Fax: + 55-11-3082-6580 \\ E-mail: mbrentani@ lim24.fm.usp.br \\ Part of this study was presented \\ at the 24th Annual San Antonio \\ Breast Cancer Symposium, December \\ 10-13, 2001.
}

Research supported by FAPESP (No. 99/12641-1).

.....................

Received March 8, 2002 Accepted June 20, 2002

\begin{abstract}
We have retrospectively analyzed a series of 155 sequential cases of T1N0M0 ductal carcinomas of which 51 tumors had a ductal carcinoma in situ (DCIS) component for correlation between the presence of DCIS and clinicopathological variables, recurrence and patient survival. No correlations between the presence of DCIS and age, menopausal status, size, estrogen or progesterone receptors were found. High-grade infiltrative tumors tended not to present a DCIS component $(\mathrm{P}=0.08)$. Patients with tumors associated with DCIS form a subgroup with few recurrences $(\mathrm{P}=0.003)$ and good survival $(\mathrm{P}=$ 0.008 ). When tumors were classified by size, an association between large tumors $(>1.0 \mathrm{~cm})$ and increased recurrence and shortened overall survival was found. The presence of DCIS in this subgroup significantly reduced the relative risk of death.
\end{abstract}

\section{Introduction}

Ductal carcinoma in situ (DCIS) is considered to be a preneoplastic lesion (1) and is increasingly identified by mammographic screening (2). It appears that DCIS comprises a heterogeneous group of lesions, the natural history of which remains unclear $(3,4)$. As a preneoplastic lesion, it shares many molecular genetic alterations with invasive carcinoma (5). DCIS can occur as a pure lesion or can be found in association with invasive carcinomas regardless of staging and extension of the disease (1). The association of DCIS with an invasive lesion is to be expected given the segmental spreading pattern described by Faverly et al. (6). While some foci of microinvasion occur and might progress, other DCIS foci in the vicinity remain in an in situ state, becoming engulfed by the infiltrative lesion. The two components represent extremes of the same carcinogenic progression and this intermediate situation thus corresponds to a distinct entity. Gupta et al. (7) proposed that invasive tumors arise from the in situ component with which they are associated and some of the biological and genetic features of the neoplastic clone in breast carcinoma may already be established at the in situ stage and 
carried through to the invasive stage of the disease. Indeed, some results support a precursor/product relationship with both components of the same tumor showing identical genetic imbalances and similar biological markers pointing to a clonal relationship between them (8-10).

Patients having breast tumors with both DCIS and an invasive component have a different prognosis than patients with invasive carcinoma without DCIS. Black et al. (11) analyzed a large series of infiltrative breast cancer that included tumors of different stages and reported that patients with invasive cancer who presented prior, simultaneous or metachronous contralateral in situ breast carcinoma had superior survival characteristics. Arisio et al. (12) analyzed a group of $\mathrm{T} 1$ carcinomas and verified that the presence of a concurrent DCIS component apparently led to reduced risk of node metastasis. In their series, however, only $34.8 \%$ of the patients presented small tumors with the absence of lymph node involvement and distant metastasis (T1N0M0). Patients with such an early diagnosed breast cancer tend to have good survival but a small percentage of them still die from their disease $(13,14)$. Although effective for large tumors, most classical prognostic factors are less reliable for small tumors and further stratification of these patients remains a challenge (15).

We considered it important, therefore, to identify features within those early lesions that might be useful for selecting patients requiring more aggressive treatment while minimizing the over-treatment in those cases bearing lesions that are unlikely to progress. We thus examined a selected retrospective series of 155 cases of T1N0M0 ductal carcinomas, 51 of which had an in situ ductal component. Tumors were classified into two groups based on the presence or absence of the concurrent DCIS component and the differences in recurrence and survival in the two groups were determined.

\section{Patients and Methods}

We studied a retrospective sequential group of paraffin-embedded samples of infiltrating ductal carcinomas classified as T1N0M0 that had been diagnosed and treated at Hospital do Câncer A.C. Camargo, São Paulo, between 1993 and 1994. Cases presenting material (surgical specimen slides) suitable for histopathological review and with clinical and survival data available were included. Any patient with evidence of the presence of other severe clinical conditions that might jeopardize outcome (such as cardiopathy, severe diabetes, renal failure, AIDS) were excluded from the study. A total of 155 cases were included and patients were treated by surgery (quadrantectomy) followed by radiotherapy. Complementary chemotherapy and hormone therapy were given to $30(19.4 \%)$ and $50(32.3 \%)$ patients, respectively. Clinical data as well as information regarding macroscopic morphology were retrieved from the patients' clinical records. Pathology slides were reviewed and reclassified for all patients. According to the information gathered from the pathology report, tumor size was divided into three categories according to the American Joint Committee on Cancer Classification (16). Invasive carcinomas were classified as T1a, when maximum tumor dimension was larger than $0.1 \mathrm{~cm}$ and less than $0.5 \mathrm{~cm}$; T1 $\mathrm{b}$, when tumors measured 0.5 to $1 \mathrm{~cm}$, and T1c when tumor dimensions were between 1 and $2 \mathrm{~cm}$. The infiltrative component of all tumors was classified using the Bloom-Richardson system (BRS). The DCIS component of the lesions was graded using the American Forces Institute of Pathology (AFIP) grading system, taking into account the presence of necrosis in the intraductal region and the nuclear grade of the lesions (17). DCIS was assessed further for the following architectural growth patterns: comedo, and noncomedo types (cribriform, micropapillary, solid and mixed types). We considered a 
DCIS component to be present in the infiltrative lesion when at least two or more foci of DCIS in different areas within the tumor were identified in the slides analyzed. The immunohistochemical expression of estrogen and progesterone receptor status was also evaluated with the following antibodies: ID5 (Dako A/S, Glostrup, Denmark) and 1.A6 (Novocastra, Newcastle, UK), respectively. A case was considered positive if more than $10 \%$ of the tumor cells were stained. Sections of female breast cancer with known results for the markers analyzed were included as negative and positive controls.

Recurrence, death from the cancer and death from other causes were determined from the hospital patient files. Associations between the presence or absence of DCIS and other clinicopathological variables were performed using the chi-square test with significance of $95 \%$. Overall survival was estimated by the Kaplan-Meier method and the log-rank test was applied to compare survival with the $95 \%$ confidence interval (18). The patients were followed up between surgery in 1993 and death or the last date of follow-up through December 2000. Cox regression was applied to estimate the relative risk (RR) and respective 95\% confidence interval (19).

\section{Results}

The DCIS component was present in 51 cases $(32.9 \%)$. The remaining 104 cases $(67.1 \%)$ consisted entirely of infiltrative lesions. Data concerning the morphologic and histological characteristics of the DCIS component are displayed in Table 1. Classification of the DCIS component present in the 51 cases showed that it was poorly or intermediately differentiated in most cases.

Table 2 illustrates the distribution of clinicopathological data according to presence or absence of DCIS in the lesions. The majority of women were white $(90.32 \%)$. Age ranged from 22 to 86 years, with a median of 55 years and a mean of 56.2 years. Fifty-two were premenopausal patients $(33.5 \%)$ and 103 postmenopausal (66.5\%). According to tumor diameter, cases were subdivided into three categories: $27 \mathrm{~T} 1 \mathrm{a}(17.4 \%), 48 \mathrm{~T} 1 \mathrm{~b}$ (31\%) and $80 \mathrm{~T} 1 \mathrm{c}(51.6 \%)$. Of the total, 66 cases $(42.6 \%)$ were estrogen receptor positive and 89 were negative (57.4\%). Progesterone receptor status was positive in 88 cases (56.8\%) and negative in 67 cases $(43.2 \%)$. The infiltrative component of all tumors was classified by the BRS system, resulting in grade I for 42 cases $(27.1 \%$ ), grade II for 73 (47.1\%) and grade III for 40 (25.8\%).

The presence of DCIS was not statistically associated with patient age or menopausal status. Neither was any association found with the histological or biochemical characteristics of the tumors. High-grade tumors tended not to present a DCIS component, although the difference was not significant $(\mathrm{P}=0.08)$. There was a weak association between the differentiation of DCIS and the grade of the invasive ductal component $(\mathrm{P}=0.06$, data not shown $)$.

Table 1. Morphological features of the ductal carcinoma in situ (DCIS) component in TINOMO breast cancers.

\begin{tabular}{lc}
\hline Histological categorization & N (\%) \\
\hline Comedo & $12(23.5)$ \\
Non-comedo & $39(76.5)$ \\
Cribriform & $13(25.4)$ \\
Micropapillary & $7(13.7)$ \\
Mixed & $5(9.8)$ \\
Solid type & $14(27.5)$ \\
Nuclear grade & \\
I & $9(17.6)$ \\
II & $29(56.8)$ \\
III & $13(25.4)$ \\
Presence of necrosis & \\
Absent & $26(51.0)$ \\
Few & $7(13.7)$ \\
Present & $18(25.4)$ \\
AFIP DCIS classification & \\
Grade I & $8(15.7)$ \\
Grade II & $23(45.1)$ \\
Grade III & $20(39.2)$
\end{tabular}


Table 2. Clinicopathological variables according to the presence or absence of ductal carcinoma in situ (DCIS) in infiltrating T1NOMO ductal carcinomas.

\begin{tabular}{|c|c|c|c|}
\hline Variable & $\begin{array}{c}\text { Number of cases } \\
\text { with DCIS }\end{array}$ & $\begin{array}{l}\text { Number of cases } \\
\text { without DCIS }\end{array}$ & $P$ \\
\hline \multicolumn{4}{|l|}{ Age (years) } \\
\hline$\leq 55$ & 28 & 50 & \\
\hline$>55$ & 23 & 54 & 0.425 \\
\hline \multicolumn{4}{|c|}{ Menopausal status } \\
\hline Pre & 20 & 32 & \\
\hline Post & 31 & 72 & 0.295 \\
\hline \multicolumn{4}{|c|}{ BRS tumor grade } \\
\hline 1 & 19 & 23 & \\
\hline II & 23 & 50 & \\
\hline III & 9 & 31 & 0.085 \\
\hline \multicolumn{4}{|c|}{ Estrogen receptor } \\
\hline Negative & 22 & 44 & \\
\hline Positive & 29 & 60 & 0.922 \\
\hline \multicolumn{4}{|c|}{ Progesterone receptor } \\
\hline Negative & 31 & 57 & \\
\hline Positive & 20 & 47 & 0.480 \\
\hline \multicolumn{4}{|c|}{ Chemotherapy } \\
\hline Yes & 10 & 20 & \\
\hline No & 41 & 84 & 0.955 \\
\hline \multicolumn{4}{|c|}{ Hormone therapy } \\
\hline Yes & 14 & 36 & \\
\hline No & 37 & 68 & 0.365 \\
\hline \multicolumn{4}{|l|}{ Recurrence } \\
\hline Yes & 2 & 24 & \\
\hline No & 49 & 80 & 0.003 \\
\hline \multicolumn{4}{|c|}{ Follow-up status } \\
\hline Alive & 50 & 86 & \\
\hline Dead & 1 & 18 & 0.006 \\
\hline \multicolumn{4}{|l|}{ Tumor size } \\
\hline $\mathrm{T} 1 \mathrm{a}+\mathrm{T} 1 \mathrm{~b}$ & 25 & 50 & \\
\hline $\mathrm{TlC}$ & 26 & 54 & 0.912 \\
\hline
\end{tabular}

BRS: Bloom-Richardson sytem. P value obtained by the chi-square test with $95 \%$ significance.

Figure 1. Kaplan-Meier sunvival estimates comparing patients presenting tumors with a concurrent in situ component (DCIS) or exhibiting pure infiltrative tumors.

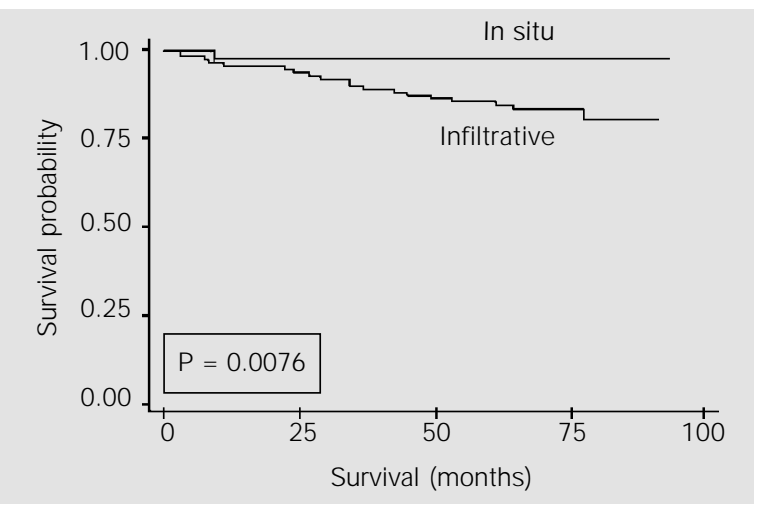

At the last follow-up, 12 patients had died of cancer $(7.74 \%)$ and 7 of other causes (4.5\%); recurrence was detected in 26 cases (16.77\%), 119 patients remained well without disease (76.7\%), and 13 (8.3\%) had evidence of disease. Four patients $(2.58 \%)$ were lost to follow-up. The mean duration of follow-up was 5.6 years (interval of 4.1 to 6 years).

As shown in Table 2, the presence of a DCIS component was negatively related to recurrence. Among the 51 cases with DCIS, only two patients relapsed (3.9\%). In contrast, in the pure infiltrative group, 24 of 104 patients had a recurrence of the tumor $(23.08 \%)$. The difference was statistically significant $(\mathrm{P}=0.003)$.

The impact of the presence of DCIS on the overall survival probability was also evaluated. None of the 12 patients who died of cancer and 6/7 that died of other causes presented a DCIS component in their lesions. Moreover, of the patients alive with disease, 11 belonged to the group with a pure infiltrative tumor while only 2 patients presented tumors with a DCIS component. Survival data are shown in Figure 1. Overall survival rate for the group of patients with an in situ component was estimated to be $98 \%$ as compared to $79.8 \%$ for the patients with pure infiltrative tumors. At 5 years the overall survival was 98 and $85.4 \%$, respectively $(\mathrm{P}=0.008)$. The BRS tumor grade had a weak prognostic value with a $97.6 \%$ overall survival rate for grade I, $76.9 \%$ for grade II and $87.5 \%$ for grade III. At 5 years, overall survival was $97.6,86.3$ and $87.5 \%$ for grade I, II and III, respectively (log-rank, P=0.053). Other histopathological variables such as presence of necrosis, desmoplasia, lymphatic vascular or perineural invasion did not influence outcome (data not shown). The influence of chemotherapy and/or hormonotherapy on survival and outcome was assessed and neither kind of treatment was an independent variable in multivariate analysis in this series. 
Our data suggest that increased tumor size also correlates with recurrence and poor survival (Table 3). Among 27 women with T1a tumors, one died of causes other than breast cancer, with no recurrence observed. Two recurrences, one breast cancer death and one death unrelated to cancer, were observed for patients with T1b tumors. Both deaths and recurrences occurred in tumors without concurrent DCIS. In T1c tumors, 54 of $80(67.5 \%)$ tumors did not have an in situ component and this group accounted for $92 \%$ (11 of 12 ) of breast cancer deaths. T1c lesions with DCIS (32.5\%) accounted for no breast cancer deaths.

To determine which factors were associated with overall survival we entered DCIS (absent versus present) and tumor size ( $\leq 1 v s$ $>1 \mathrm{~cm}$ ) into a Cox regression univariate and multivariate analysis (Table 4). Tumor size was significantly associated with poor survival, with a crude RR of 5.39 (1.57-1.85) and a risk of death of $5.65(1.65-19.44)$ in multivariate analysis. The association with DCIS showed a greatly reduced risk of death. With a DCIS present, there was a decrease in the crude RR and in RR (multivariate) when adjusted to tumor size.

\section{Discussion}

Few studies have focused on the combination of infiltrating and DCIS components in breast ductal carcinomas. Previous analyses of the frequency of well-differentiated DCIS associated with infiltrating ductal carcinomas found this to be present in less than $10 \%(20,21)$. We found $15.7 \%$ of AFIP grade I DCIS in our specific series of T1N0M0 tumors, which is less than the 21 to $45 \%$ value found in other studies of welldifferentiated DCIS in pure DCIS lesion series $(6,22)$. One possible explanation is that well-differentiated DCIS does not often progress to invasion or that the subsequent tumor is likely to be more differentiated and probably associated with a good prognosis
$(20,23)$. In this context, Buerger et al. (24) have demonstrated the presence of the same genetic aberrations, namely $16 \mathrm{q}$ loss and $1 \mathrm{q}$ gain, in well-differentiated DCIS lesions and tubular and tubulolobular carcinomas with a good prognosis. In our study of 51 cases, recurrence was observed in only two patients neither of whom was classified as AFIP grade I.

It has been reported that the grade of the DCIS component corresponds to the infiltrative carcinoma grading $(7,10,20)$. We found only a slightly positive association $(\mathrm{P}=0.06)$ between DCIS and infiltrative carcinoma grade, in agreement with one other study (21). In agreement with the report by Tabár et al. (15), malignancy grade was a weak

Table 3. Relationship between tumor size and follow-up status of 155 patients with infiltrating T1NOM0 ductal carcinomas.

\begin{tabular}{lccc}
\hline Variable & \multicolumn{2}{c}{ Tumor size } & $\mathrm{P}$ \\
\cline { 2 - 3 } & Tla $+\mathrm{Tlb}$ & $\mathrm{Tlc}$ & \\
\hline Recurrence & & & \\
Yes & 2 & 24 & $<0.001$ \\
No & 73 & 56 & \\
Death & & & \\
Yes & 3 & 16 & 0.002 \\
No & 72 & 64 & \\
Total & 75 & 80 & \\
\hline
\end{tabular}

Tumors were categorized according to the American J oint Committee on Cancer Classification (Ref. 16). Invasive carcinomas were classified as $\mathrm{Tla}$ $\leq 5 \mathrm{~mm}, \mathrm{Tl} b=6-10 \mathrm{~mm}$ and $\mathrm{Tlc}=11-20 \mathrm{~mm}$.

Table 4. Relative risk (RR) and respective $95 \%$ confidence interval (CI) estimated by Cox regression (Ref. 19) analysis for ductal carcinoma in situ (DCIS) and tumor size.

\begin{tabular}{lcccccc}
\hline Variable & $\begin{array}{c}\text { Crude } \\
\text { RR }\end{array}$ & $95 \%$ Cl & P & $\begin{array}{c}\text { Multivariate } \\
\text { RR }\end{array}$ & $95 \% \mathrm{Cl}$ & P \\
\hline Without DCIS & 1.00 & $*$ & & 1.00 & $*$ & \\
With DCIS & 0.11 & $0.01-0.79$ & 0.029 & 0.10 & $0.01-0.75$ & 0.026 \\
Tla + Tlb & 1.00 & $*$ & & 1.00 & $*$ & \\
Tlc & 5.39 & $1.57-18.51$ & 0.007 & 5.65 & $1.64-19.44$ & 0.006
\end{tabular}

*Reference categories. See legend to Table 3 for tumor size classification. 
predictor of survival in our series.

We have shown that tumor size in T1N0M0 breast cancer is a reliable determinant of recurrence rate and survival. In our series, almost all recurrences occurred in tumors larger than $1.0 \mathrm{~cm}$ in diameter (T1c). Survival also improved as tumor size decreased. These results confirm previous reports (12-15). Although size remains an important prognostic factor in T1N0M0 tumors, the novel aspect of our study is the possibility of further stratification of less favorable T1c cases. Our data indicate that DCIS is a variable that positively reduces the impact of size on survival.

Our finding that identification of a DCIS component concurrent with invasive carcinomas in a well-defined cohort of tumors confers a significantly better prognosis to patients is in accordance with previous studies analyzing more heterogeneous series of breast cancer $(11,12)$. Black et al. (11) suggested that an in situ carcinoma-associated immunogenicity might partially explain the more favorable survival of these patients. Another possible explanation is that the favorable biological characteristics of DCIS are maintained in the cells of the same clonal populations, conferring a less aggressive phenotype to the associated infiltrative carcinoma (7).

Our results are consistent with the concept that, in general, tumors originating from in situ lesions are less aggressive than entirely invasive tumors $(9,12,25,26)$.

\section{References}

1. Lakhani SR (1999). The transition from hyperplasia to invasive carcinoma of the breast. J ournal of Pathology, 187: 272278.

2. Rajakariar R \& Walker RA (1995). Pathological and biological features of mammographically detected invasive breast carcinomas. British J ournal of Cancer, 71: 150154.

3. Page DL \& J ensen RA (1996). Ductal carcinoma in situ of the breast: understanding the misunderstood stepchild. J oumal of the American Medical Association, 275: 948-949.

4. Welch HG \& Black WC (1997). Using autopsy series to estimate the disease "reservoir" for ductal carcinoma in situ of the breast: how much more breast cancer can we find? Annals of Internal Medicine, 127: 1023-1028.

5. Stratton MR, Collins N, Lakhani SR \& Sloane JP (1995). Loss of heterozygosity in ductal carcinoma in situ of the breast. J oumal of Pathology, 175: 195-201.

6. Faverly DR, Burgers L, Bult P \& Holland R (1994). Three dimensional imaging of ductal carcinoma in situ: Clinical implications. Seminars in Diagnostic Pathology, 11: 193-198.

7. Gupta SK, Douglas-J ones AG, Fenn N, Morgan J M \& Mansel RE (1997). The clinical behavior of breast carcinoma is probably determined at the preinvasive stage (ductal carcinoma in situ). Cancer, 80: 1740-1745.

8. Allred DC, Clark GM, Molina R, Tandon AK, Schnitt SJ , Gilchrist KW, Osbome CK, Tormey DC \& McGuire WL (1992). Overexpression of HER-2/neu and its relationship with other prognostic factors change during the progression of in situ to invasive breast cancer. Human Pathology, 23: 974-979.

9. Lukas J, Niu N \& Press MF (2000). p53 mutations and expression in breast carcinoma in situ. American J ournal of Pathology, 156: 183-191.

10. Leong AS-Y, Sormunen RT, Vinyuvat S, Hamdani RW \& Suthipintawong C (2001). Biologic markers in ductal carcinoma in situ and concurrent infiltrating carcinoma. A comparison of eight contemporary grading systems. American J oumal of Clinical Pathology, 115: 709-718.

11. Black MM, Zachrau RE, Hankey BF \& Feuer EJ (1996). Prognostic significance of in situ carcinoma associated with invasive breast carcinoma. Cancer, 78: 778788.

12. Arisio R, Sapino A, Cassoni P, Accinelli G, Ciccorese MC, Mano MP \& Bussolati G (2000). What modifies the relation between tumour size and lymph node metastases in $\mathrm{T} 1$ breast carcinomas? J ournal of Clinical Pathology, 53: 846-850.

13. Tabár L, Duffy SW, Vitak $B \&$ Chen $\mathrm{H}-\mathrm{H}$
(1999). The natural history of breast carcinoma: What have we learned from screening? Cancer, 86: 449-462.

14. J oensuu $H$, Pylkänen $L \&$ Toikkanen $S$ (1999). Late mortality from pTINOMO breast carcinoma. Cancer, 85: 2183-2189.

15. Tabár L, Chen HH, Duffy SW, Yen MF, Chaing CF, Dean PB \& Smith RA (2000). A novel method for prediction of long-term outcome of women with Tla, Tlb, and 10-14 mm invasive breast cancers: a prospective study. Lancet, 355: 429-433.

16. Sobin LH \& Wittekindch TNM (1997). Classification of Malignant Tumours. 5th edn. Wiley-Liss, New York, NY, USA.

17. Tavassoli FA \& Man YG (1995). Morphofunctional features of intraductal hyperplasia, atypical intraductal hyperplasia, and various grades of intraductal carcinoma. Breast J oumal, 1: 155-162.

18. Kaplan EL \& Meier P (1958). Nonparametric estimation from incomplete observations. J ournal of the American Statistical Association, 53: 457-481.

19. Cox DR (1972). Regression models and life-tables. J oumal of the Royal Statistical Society, 34: 187-220.

20. Lampejo OT, Bames DM, Smith $P \&$ Millis RR (1994). Evaluation of infiltrating ductal carcinomas with a DCIS component: correlation of the histologic type of the in situ component with grade of the infiltrating component. Seminars in Diagnostic Pa- 
thology, 11: 215-222.

21. Goldstein NS \& Murphy T (1996). Intraductal carcinoma associated with invasive carcinoma of the breast. A comparison of the two lesions with implications for intraductal carcinoma classification systems. American J ournal of Clinical Pathology, 106: 312-318.

22. Bobrow LG, Happerfield LC, Gregory WM, Springall RD \& Millis RR (1994). The classification of ductal carcinoma in situ and its association with biological markers. Seminars in Diagnostic Pathology, 11:
199-207.

23. Eusebi V, Feudale E, Foschini MP, Micheli A, Conti A, Riva C, Di Palma S \& Rilke F (1994). Long-term follow-up of in situ carcinoma of the breast. Seminars in Diagnostic Pathology, 11: 223-235.

24. Buerger $H$, Otterbach $F$, Simon $R$, Schafer K-L, Poroemba C, Diallo R, Brinksmidt C, Dickhorn-Dworniczak B \& Boecker W (1999). Different genetic pathways in the evolution of invasive breast cancer are associated with distinct morphological subtypes. J oumal of Pathology, 189: 521-526.
25. Querzoli P, Albonico G, Ferretti S, Rinaldi R, Becatti D, Corcione S, Indelli M \& Nenci I (1998). Modulation of biomarkers in minimal breast carcinoma. Cancer, 83: 89-97.

26. Barnes DM, Bartkova J, Camplejohn RS, Gullick WJ , Smith PJ \& Millis RR (1992). Overexpression of the c-erbB-2 oncoprotein: Why does this occur more frequently in ductal carcinoma in situ than in invasive mammary carcinoma and is this of prognostic significance? European J ournal of Cancer, 28: 644-648. 


\section{THE NOVARTIS FOUNDATION BURSARY SCHEME}

promoting excellence in science worldwide
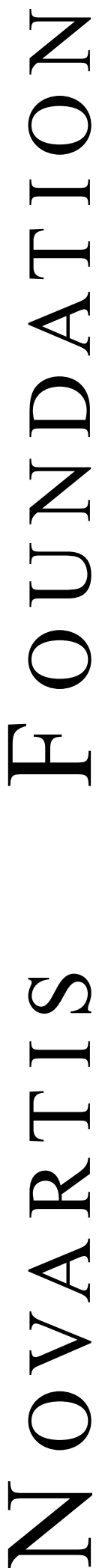

\section{INTRODUCTION}

The Novartis Foundation bursary scheme is linked to the Novartis Foundation symposia. This scheme enables one young scientist to attend a Novartis Foundation symposium (in London except where otherwise specified) and immediately following the meeting to spend a period in the laboratory of one of the participants.

\section{DURATION AND FORMAT}

The bursary is granted for a period of up to three months which includes travel, attendance at a Novartis symposium and from four to twelve weeks visiting a participant's laboratory. The actual period will be agreed between the host and bursar.

\section{BURSARS}

Bursars must be aged 23-35 and actively engaged in research in the field in question. They should not already have accepted an invitation to participate in that symposium. Successful candidates are expected to submit a short report following their return home.

\section{PROCEDURE}

Making the bursaries known: The availability of the bursaries is advertised by circular to overseas members of the Foundation's Scientific Advisory Panel, to invited symposiasts and by an advertisement in Nature, or other journal if more appropriate. Bursaries are advertised every 3-6 months, and at least six months before the date of the relevant meeting.

Applying: We do not issue application forms. Written applications should be made to the Bursary Scheme Administrator at the Novartis Foundation, including the following information:

- Full name, address, birthdate

- Title of symposium for which applying

- Qualifications and short resumé of further education
- Career history, including full list of publications

- Full details of current research

- Aims of future career

- Name \& address of two referees

Selection of the bursar: This is made by the Foundation's senior staff, usually around four months before the symposium.

Which laboratory/department? Offers to host a bursar are sought from symposiasts at the time of invitation to the symposium. The successful bursar is asked to select three names from the membership list of the symposium and every effort is made by the Novartis Foundation to accommodate the bursar's choice.

\section{VALUE OF THE BURSARY}

The bursary covers the following:

- All the bursar's travel expenses using the cheapest, most practicable and direct means of travel (i.e. economy class fares using APEX fares whenever possible).

- Bed and breakfast accommodation during the symposium week, with an allowance for meals.

Board and lodging during the candidate's visit to the host laboratory. It is expected that the bursar will be able to stay in university accommodation or, if that is not available, in a modestly priced hotel.

\section{CONTACT}

Novartis Foundation Bursary Scheme Administrator, 41 Portland Place, London W1N 4BN, UK. E-mail: bursary@,novartisfound.org.uk 